\title{
Accurate Time-Varying Sources in $k$-Space Pseudospectral Time Domain Acoustic Simulations
}

\author{
B.T. Cox \& B.E. Treeby \\ Department of Medical Physics and Biomedical Engineering, University College London, UK \\ b.cox@ucl.ac.uk
}

\begin{abstract}
The pseudospectral time domain (PSTD) method is popular as a wave equation solver, one of its advantages being that it permits the use of coarse grids, as long as the spatial sampling is greater than two points-per-wavelength. The $k$-space PSTD method improves on this by incorporating an additional factor to remove the numerical dispersion which arises from the discretisation of the time derivatives. However, the effect of the temporal discretisation of time-varying sources has not previously been considered, although it can lead to substantial errors in the predicted field unless the timestep is sufficiently small to ensure the highest frequency is sampled at many points-per-period. Here, a correction factor is derived which eliminates this source of error, resulting in a $k$-space PSTD method that retains the spatial sampling advantage of conventional PSTD schemes but is also accurate as long as the temporal sampling is greater than two points-per-period and the CFL number is $<1$.

Index Terms-acoustic simulation, pseudospectral time domain, k-space, sources
\end{abstract}

\section{INTRODUCTION}

The $k$-space pseudospectral time domain (PSTD) method [1]-[4] is a time-stepping scheme for full-wave acoustic simulations. It is widely used, for example in the $k$-Wave implementation [5], because of its computational efficiency. Spatial gradients are computed using a spectral method, which introduces no approximation errors as long as the computational grid-spacing is less than half the shortest wavelength (Nyquist), thereby minimizing the computational memory required for simulations. Temporal gradients are computed using forward differences, but the numerical dispersion that would result from this approximation is eliminated through the use of a correction factor applied in the wavenumber domain or $k$-space. When solving initial value problems in homogeneous media, this method can calculate the acoustic field exactly at any future time, ie. there are no restrictions on the size of the timestep that can be taken. This comes as no surprise when one learns that the correction factor is derived directly from the free-space Green's function.

For problems involving time-varying sources, however, there remains a potential source of error. While a correction factor which mitigates for the temporal discretisation of the time derivatives is incorporated in the scheme, no such factor is included to account for the temporal discretisation of the

This work was supported by the European Union's Horizon 2020 research and innovation program H2020 ICT 2016-2017 under Grant agreement No. 732411, which is an initiative of the Photonics Public Private Partnership, and by the Engineering and Physical Sciences Research Council, UK. source terms. Of course, the method is nevertheless convergent: for smaller and smaller timesteps, ie. higher and higher sampling rates of the source function, the solution approaches ever more closely the true solution. However, the requirement to take small timesteps in order to model a time-varying source accurately feels out-of-place in the context of a $k$-space PSTD method that only requires spatial sampling be better than Nyquist. In this paper, a $k$-space source correction factor is derived from a Green's function solution. This correction removes the error due to the temporal sampling of the source function, resulting in a scheme which also only requires the temporal sampling be better than Nyquist.

\section{II. $k$-SPACE PSEUDOSPECTRAL TIME DOMAIN MODEL: FIRST-ORDER SYSTEM}

The wave equation for the acoustic pressure $p$ in a homogeneous medium with sound speed $c_{0}$ and density $\rho_{0}$ is

$$
\left(\nabla^{2}-c_{0}^{-2} \partial_{t t}\right) p=\rho_{0} \nabla \cdot \mathbf{F}-\partial_{t} M
$$

where $\mathbf{F}=\left(F_{x}, F_{y}\right)$ is a vector force source term and represents the input of body forces per unit mass in units of $\mathrm{N} . \mathrm{kg}^{-1}$ or $\mathrm{m} . \mathrm{s}^{-2}$ and $M$ is a mass source term and represents the time rate of the input of mass per unit volume in units of $\mathrm{kg} \cdot \mathrm{m}^{-3} \mathrm{~s}^{-1}$. (The $2 \mathrm{D}$ case will be described in this paper for conciseness but the extension to $3 \mathrm{D}$ is straightforward.) This can be written as a system of first-order equations:

$$
\partial_{t} \mathbf{u}=-\nabla p / \rho_{0}+\mathbf{F}, \quad \partial_{t} \rho=-\rho_{0} \nabla \cdot \mathbf{u}+M, \quad p=c_{0}^{2} \rho,
$$

where $\mathbf{u}=\left(u_{x}, u_{y}\right)$ and $\rho$ are the acoustic particle velocity and density. Writing the time derivatives as finite differences suggests the following time-stepping scheme:

$$
\begin{aligned}
& u_{x}^{n+\frac{1}{2}}=u_{x}^{n-\frac{1}{2}}-\Delta t \partial_{x} p^{n} / \rho_{0}+\Delta t F_{x}^{n}, \\
& u_{y}^{n+\frac{1}{2}}=u_{y}^{n-\frac{1}{2}}-\Delta t \partial_{y} p^{n} / \rho_{0}+\Delta t F_{y}^{n}, \\
& \rho^{n+1}=\rho^{n}-\Delta t \rho_{0}\left(\partial_{x} u_{x}^{n+\frac{1}{2}}+\partial_{y} u_{y}^{n+\frac{1}{2}}\right)+\Delta t M^{n+\frac{1}{2}}, \\
& p^{n+1}=c_{0}^{2} \rho^{n+1},
\end{aligned}
$$

where the superscript $n$ indicates the value at the $n^{\text {th }}$ timestep, ie. at time $t=n \Delta t$. In the $k$-space PSTD method, the numerical dispersion that would arise from the finite differences is corrected exactly by a factor [2]:

$$
\kappa=\operatorname{sinc}\left(c_{0} k \Delta t / 2\right),
$$


where $k=|\mathbf{k}|$ is the wavenumber, the magnitude of the wavevector. Because the correction factor $\kappa$ needs to be applied in $k$-space, it is convenient to add it to the gradient calculation, giving:

$$
\begin{aligned}
\partial_{x} u_{x}^{n+\frac{1}{2}} & =\mathcal{F}^{-1}\left\{i k_{x} \kappa \mathcal{F}\left\{u_{x}^{n+\frac{1}{2}}\right\}\right\}, \\
\partial_{x} p^{n} & =\mathcal{F}^{-1}\left\{i k_{x} \kappa \mathcal{F}\left\{p^{n}\right\}\right\},
\end{aligned}
$$

and similar expressions for the gradients in the $y$ direction. Here $\mathcal{F}$ indicates a Fourier transform, and $k_{x}$ and $k_{y}$ are the components of the wavevector $\mathbf{k}$. Equations (2)-(8) form a convergent numerical scheme, meaning that for short enough timesteps it will converge to the solution of (1). Furthermore, when solving the initial value problem $\left(\nabla^{2}-c_{0}^{-2} \partial_{t t}\right) p=0$ with initial conditions for $p$ and $\partial_{t} p$, the use of $\kappa$ means that this model will give the exact solution for any size timestep. However, for a time-varying source this is not generally true, as the temporal discretisation of the source has not been taken explicitly into account. This acoustic model will therefore be referred to as the uncorrected scheme.

This paper shows that this scheme can be improved for time-varying sources by finding a correction term which, when applied to the source terms in Eqs. (2)-(4), ensures the exactness of the solutions. Such a correction is limited in principle to timesteps smaller than half the period of the highest frequency in the source, in the same way the grid spacing must be less than half the shortest wavelength (although in practice 3 points-per-wavelength are typically required and the Courant-Friedrichs-Lewy number (CFL) must be $<1$ ). In general, time-varying sources will be broadband, but because any signal can be written as a sum of sinusoids, it is necessary only to derive the correction factor for a single frequency, and the case of a broadband source will come from it. The approach taken here will therefore be to compare the uncorrected scheme to a $k$-space PSTD model derived from an exact solution of Eq. (1) for single frequency sources. First, though, to facilitate this comparison, the uncorrected scheme will be rewritten in second-order form.

\section{III. $k$-SPACE PSTD MODEL: SECOND-ORDER FORM}

To determine whether correction factors are required for the source terms in the uncorrected scheme given in Eqs. (2)-(4), the first-order scheme that they represent will be rearranged into a second-order scheme so that it can be compared with the exact scheme to be derived below. Combining Eqs. (4), (5) and (7) gives, in $k$-space:

$$
\begin{aligned}
\hat{p}^{n+1}=\hat{p}^{n} & -\Delta t c_{0}^{2} \rho_{0}\left(i k_{x} \kappa \hat{u}_{x}^{n+\frac{1}{2}}+i k_{y} \kappa \hat{u}_{y}^{n+\frac{1}{2}}\right) \\
& +\Delta t c_{0}^{2} \hat{M}^{n+\frac{1}{2}}
\end{aligned}
$$

where $\hat{~}$ indicates a variable in $k$-space, eg. $\hat{p}(\mathbf{k})=\mathcal{F}\{p(\mathbf{x})\}$. Writing a similar expression for the pressure at the previous timestep $\hat{p}^{n}$, and subtracting $\hat{p}^{n}$ from $\hat{p}^{n+1}$, gives

$$
\begin{aligned}
& \hat{p}^{n+1}-2 \hat{p}^{n}+\hat{p}^{n-1}=\Delta t c_{0}^{2} \rho_{0} \times \\
& \left(-\left(i k_{x} \kappa \hat{u}_{x}^{n+\frac{1}{2}}+i k_{y} \kappa \hat{u}_{y}^{n+\frac{1}{2}}\right)+\left(i k_{x} \kappa \hat{u}_{x}^{n-\frac{1}{2}}+i k_{y} \kappa \hat{u}_{y}^{n-\frac{1}{2}}\right)\right) \\
& +\Delta t c_{0}^{2}\left(\hat{M}^{n+\frac{1}{2}}-\hat{M}^{n-\frac{1}{2}}\right) .
\end{aligned}
$$

Substituting in the $k$-space versions of Eqs. (2) and (3) gives

$$
\begin{aligned}
\hat{p}^{n+1}-2 \hat{p}^{n} & +\hat{p}^{n-1}=\Delta t c_{0}^{2} \rho_{0} \times \\
( & -i k_{x} \kappa\left(\hat{u}_{x}^{n-\frac{1}{2}}-\Delta t i k_{x} \kappa \hat{p}^{n} / \rho_{0}+\Delta t \hat{F}_{x}^{n}\right) \\
& -i k_{y} \kappa\left(\hat{u}_{y}^{n-\frac{1}{2}}-\Delta t i k_{y} \kappa \hat{p}^{n} / \rho_{0}+\Delta t \hat{F}_{y}^{n}\right) \\
& \left.+\left(i k_{x} \kappa \hat{u}_{x}^{n-\frac{1}{2}}+i k_{y} \kappa \hat{u}_{y}^{n-\frac{1}{2}}\right)\right) \\
& +\Delta t c_{0}^{2}\left(\hat{M}^{n+\frac{1}{2}}-\hat{M}^{n-\frac{1}{2}}\right) .
\end{aligned}
$$

Cancelling terms leaves

$$
\begin{aligned}
\frac{\hat{p}^{n+1}-2 \hat{p}^{n}+\hat{p}^{n-1}}{\Delta t^{2}}= & -\left(c_{0} k \kappa\right)^{2} \hat{p}^{n} \\
& -c_{0}^{2} \kappa \rho_{0}\left(i k_{x} \hat{F}_{x}^{n}+i k_{y} \hat{F}_{y}^{n}\right) \\
& +c_{0}^{2} \kappa\left(\hat{M}^{n+\frac{1}{2}}-\hat{M}^{n-\frac{1}{2}} /(\kappa \Delta t)\right)
\end{aligned}
$$

The first line of this rearrangement of the uncorrected scheme is a $k$-space pseudospectral model of the wave equation in the absences of sources, the second line includes velocity sources, and the third the mass sources (written in the form of an exact finite difference [7]).

\section{IV. $k$-SPACE PSTD MODEL: EXACT CW SOURCE TERMS}

In this section, a PSTD scheme will be derived without approximation from an exact solution to Eq. (1) in the case of single frequency sources. This will then be compared to Eq. (12) to determine if any correction factors are required in the latter. In the continuous wave case in which the sources emit sound waves of a single frequency, $\omega_{0}$ rads.s ${ }^{-1}$, the two source types can be written in separable form with complex spatially-varying amplitudes:

$$
\begin{aligned}
M(\mathbf{x}, t) & =A(\mathbf{x}) e^{i \omega_{0} t} \\
\mathbf{F}(\mathbf{x}, t) & =\left(B_{x}(\mathbf{x}), B_{y}(\mathbf{x})\right) e^{i \omega_{0} t} .
\end{aligned}
$$

The solution to Eq. (1), assuming zero initial conditions and free-space boundary conditions, can be written as [6]

$$
p(\mathbf{x}, t)=\frac{c_{0}^{2}}{(2 \pi)^{N}} \iint S\left(\mathbf{x}^{\prime}\right) I(\mathbf{k}, t) e^{i \mathbf{k} \cdot\left(\mathbf{x}-\mathbf{x}^{\prime}\right)} d \mathbf{k} d \mathbf{x}^{\prime},
$$

where $\mathbf{k}=\left(k_{x}, k_{y}\right)$ is the wavevector, the sources

$$
S\left(\mathbf{x}^{\prime}\right)=i \omega A-\rho_{0} \nabla \cdot\left(B_{x}, B_{y}\right),
$$

and the convolution of the Green's function and time factor gives [6]

$$
I(\mathbf{k}, t)=\frac{c_{0} k\left(e^{i \omega_{0} t}-\cos \left(c_{0} k t\right)\right)-i \omega_{0} \sin \left(c_{0} k t\right)}{\left(c_{0} k\right)^{3}-c_{0} k \omega_{0}^{2}} .
$$


In k-space, the pressure can be written

$$
\hat{p}(\mathbf{k}, t)=c_{0}^{2}\left(i \omega \hat{A}(\mathbf{k})-\rho_{0}\left(i k_{x} \hat{B}_{x}(\mathbf{k})+i k_{y} \hat{B}_{y}(\mathbf{k})\right)\right) I(\mathbf{k}, t) .
$$

The aim is to derive a second-order PSTD model, so, to that end, and using the notation $\hat{p}^{n+1}=\hat{p}(\mathbf{k}, t+\Delta t)$, etc. we write:

$$
\begin{aligned}
& \hat{p}^{n+1}-2 \hat{p}^{n}+\hat{p}^{n-1}= \\
& c_{0}^{2}\left(i \omega \hat{A}-\rho_{0}\left(i k_{x} \hat{B}_{x}+i k_{y} \hat{B}_{y}\right)\right)\left(I^{n+1}-2 I^{n}+I^{n-1}\right) .
\end{aligned}
$$

Some rearranging gives

$$
\begin{gathered}
I^{n+1}-2 I^{n}+I^{n-1}=-4 I^{n} \sin ^{2}\left(c_{0} k \Delta t / 2\right) \\
+\frac{4 e^{i \omega_{0} t}\left(\sin ^{2}\left(c_{0} k \Delta t / 2\right)-\sin ^{2}\left(\omega_{0} \Delta t / 2\right)\right)}{\left(c_{0} k\right)^{2}-\omega_{0}^{2}}
\end{gathered}
$$

Substituting this back into Eq. (18) and rearranging gives

$$
\begin{aligned}
& \frac{\hat{p}^{n+1}-2 \hat{p}^{n}+\hat{p}^{n-1}}{\Delta t^{2}}=-\left(c_{0} k \kappa\right)^{2} \hat{p}^{n} \\
& +c_{0}^{2}\left(i \omega \hat{A}-\rho_{0}\left(i k_{x} \hat{B}_{x}+i k_{y} \hat{B}_{y}\right)\right) e^{i \omega_{0} t} \mathcal{C}\left(k, \omega_{0}, t\right)
\end{aligned}
$$

where $\kappa$ is given in Eq. (6) and

$$
\mathcal{C}\left(k, \omega_{0}, t\right)=\frac{2\left(\cos \left(\omega_{0} \Delta t\right)-\cos \left(c_{0} k \Delta t\right)\right)}{\Delta t^{2}\left(\left(c_{0} k\right)^{2}-\omega_{0}^{2}\right)}
$$

No approximations have been made in deriving Eq. (20), which is therefore a second-order $k$-space PSTD model which is exact for homogeneous media and single-frequency sources.

\section{BROADBAND SOURCE TERM CORRECTION FACTOR}

The two second-order models given in Eqs. (12) and (20) can now be compared to see whether the former requires source corrections. In summary, the source terms in the uncorrected scheme are

$$
\begin{aligned}
& \text { mass source }=c_{0}^{2} \kappa\left(\hat{M}^{n+\frac{1}{2}}-\hat{M}^{n-\frac{1}{2}}\right) /(\kappa \Delta t), \\
& \text { force source }=-c_{0}^{2} \rho_{0} \kappa\left(i k_{x} \hat{F}_{x}^{n}+i k_{y} \hat{F}_{y}^{n}\right),
\end{aligned}
$$

and for the exact scheme are

$$
\begin{aligned}
& \text { mass source }=c_{0}^{2} \mathcal{C} i \omega_{0} \hat{M}, \\
& \text { force source }=-c_{0}^{2} \rho_{0} \mathcal{C}\left(i k_{x} \hat{F}_{x}+i k_{y} \hat{F}_{y}\right) .
\end{aligned}
$$

Comparing these source terms shows they differ by a factor

$$
\sigma=\frac{\mathcal{C}}{\kappa}=\frac{2\left(\cos \left(\omega_{0} \Delta t\right)-\cos \left(c_{0} k \Delta t\right)\right)}{\Delta t^{2} \operatorname{sinc}\left(c_{0} k \Delta t / 2\right)\left(\left(c_{0} k\right)^{2}-\omega_{0}^{2}\right)} .
$$

As the factor $\mathcal{C}$ was derived for the single frequency case, $\sigma$ can be expressed in two other forms by taking the limits

$$
\begin{aligned}
& k \rightarrow \omega_{0} / c_{0}, \quad \sigma \rightarrow \cos \left(\omega_{0} \Delta t / 2\right), \\
& \omega_{0} \rightarrow c_{0} k, \quad \sigma \rightarrow \cos \left(c_{0} k \Delta t / 2\right) .
\end{aligned}
$$

These expressions are equivalent in the continuous setting, but will have slightly different effects in the numerical case due to the regular (Cartesian) sampling of $k$-space. Also, which is more useful in practice will depend on whether the source is single-frequency or broadband. Eq. (27) gives a simple scalar amplitude scaling factor for the source but relies on knowledge of the source frequency, whereas Eq. (28) can be applied to broadband sources without needing to specify the frequency but requires two additional spatial FFTs per time step. All the analysis up to this point has assumed a single frequency source, but Eq. (28) makes no reference to the source frequency so can be applied in the broadband case, when the temporal parts of Eqs. (13) and (14) are not at a single frequency but are a Fourier sum of many frequency components. Including the broadband correction factor, Eq. (28), in the first order equations gives the following scheme, which will be referred to as the source-corrected scheme:

$$
\begin{aligned}
& \partial_{x} p^{n}=\mathcal{F}^{-1}\left\{i k_{x} \kappa \mathcal{F}\left\{p^{n}\right\}\right\}, \\
& \partial_{y} p^{n}=\mathcal{F}^{-1}\left\{i k_{y} \kappa \mathcal{F}\left\{p^{n}\right\}\right\}, \\
& u_{x}^{n+\frac{1}{2}}=u_{x}^{n-\frac{1}{2}}-\Delta t \partial_{x} p^{n} / \rho_{0}+\Delta t \mathcal{F}^{-1}\left\{\sigma \mathcal{F}\left\{F_{x}^{n}\right\}\right\}, \\
& u_{y}^{n+\frac{1}{2}}=u_{y}^{n-\frac{1}{2}}-\Delta t \partial_{y} p^{n} / \rho_{0}+\Delta t \mathcal{F}^{-1}\left\{\sigma \mathcal{F}\left\{F_{y}^{n}\right\}\right\}, \\
& \partial_{x} u_{x}^{n+\frac{1}{2}}=\mathcal{F}\left\{i k_{x} \kappa \mathcal{F}\left\{u_{x}^{n+\frac{1}{2}}\right\}\right\}, \\
& \partial_{y} u_{y}^{n+\frac{1}{2}}=\mathcal{F}\left\{i k_{y} \kappa \mathcal{F}\left\{u_{y}^{n+\frac{1}{2}}\right\}\right\}, \\
& \rho^{n+1}=\rho^{n}-\Delta t \rho_{0}\left(\partial_{x} u_{x}^{n+\frac{1}{2}}+\partial_{y} u_{y}^{n+\frac{1}{2}}\right)+\sigma \Delta t M^{n+\frac{1}{2}}, \\
& p^{n+1}=c_{0}^{2} \rho^{n+1} .
\end{aligned}
$$

\section{NUMERICAL EXAMPLE}

A numerical example illustrating the impact of the source correction is shown in Fig. 1. This was simulated using the function kspaceFirstorder3D from the open-source $k$ Wave toolbox. This implementation of the $k$-space PSTD method was modified to run with and without the source correction. (This feature will be included in the next release of $k$-Wave.) The source geometry was defined as a circular piston with a $5 \mathrm{~mm}$ radius using the approach described in [8]. The source was assigned as a mass source and driven simultaneously at $0.5 \mathrm{MHz}$ and $1 \mathrm{MHz}$ with a pressure of $1 \mathrm{MPa}$. The grid spacing was set at 3 points-per-wavelength and the timestep chosen to give 4 points-per-period at $1 \mathrm{MHz}$ (resulting in a CFL of 0.75). The source correction, Eq. (28), was applied by taking an FFT of the source matrix at each time step, applying the source correction, and then taking an inverse FFT before adding it to the matrix of pressure values, as in the source-corrected scheme above. For reference, the analytical solution for the axial pressure was calculated using Eq. 57.3 in [9]. As seen in Fig. 1(a), when no source correction is applied, there is a large error in the pressure amplitude. It is instructive to note that in the limit that the number of points-per-wavelength and points-per-period approach 2, this error grows without bound. (This can be seen by noting that the correction factor, $\sigma$, which exactly counteracts this error, shrinks to zero in the same limit.) Fig. 1(b) shows that this error decreases with increasing temporal sampling (decreasing CFL) although at the cost of increased compute time. However, in contrast, when the source correction is used, the error is as small at 4 points-per-period as at 50, and, as Fig. 1(a) shows, the axial response is very close to the analytical solution. Any residual error is due to the perfectly matched 

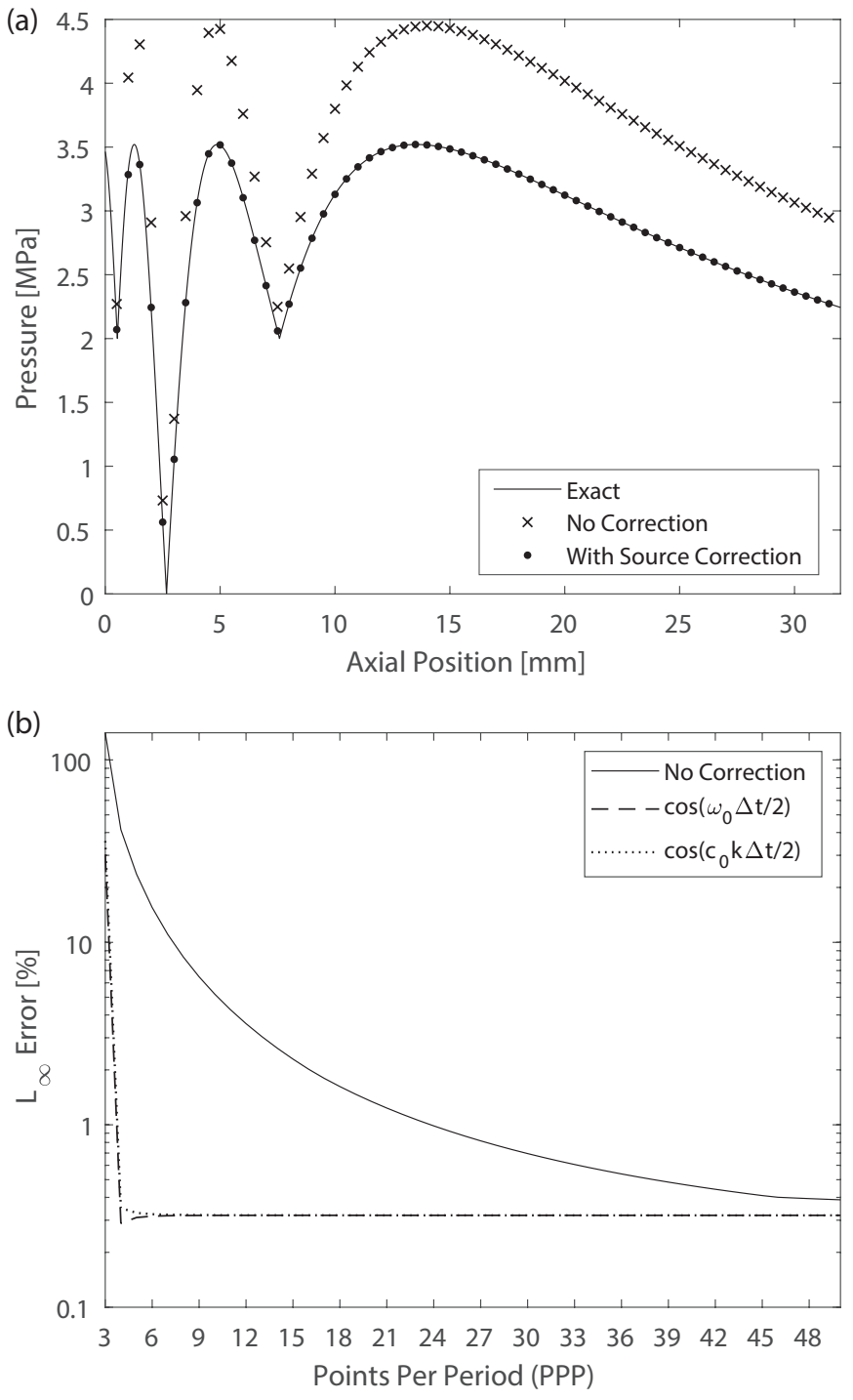

Fig. 1. Numerical example showing the effect of the source correction, (28), on the on-axis pressure field from a $5 \mathrm{~mm}$ radius circular piston source driven simultaneously at $0.5 \mathrm{MHz}$ and $1 \mathrm{MHz}$. (a) The magnitude of the on-axis pressure. The numerical solutions were calculated using $k$-Wave, with and without the source correction, using a CFL number of 0.75 (PPW $=3$, PPP $=4$ ). The solid line shows the analytical solution. (b) The maximum value ( $L_{\infty}$ norm) of the error as a function of the points-per-period at the highest frequency (temporal sampling rate).

layer absorbing boundary conditions - not described in the equations above [2], [3] - and the error in the discretisation of the source geometry. The error is larger at 3 points-per-period because in this simulation that corresponds to reaching a CFL number of 1. Fig. 1(b) shows the error when using the source correction in Eq. (27) as well as for the source correction in Eq. (28). The former was applied frequency-by-frequency to the source function before running the simulation, the latter (which was used in Fig. 1(a)) was applied every timestep during the simulation, resulting in an additional 2 spatial FFTs per timestep. There is very little difference between the error in the two cases, as expected.

\section{CONCLUSION}

A $k$-space pseudospectral time domain scheme for solving the wave equation was derived. In conventional $k$-space PSTD schemes, the spatial part of the source terms can be described without error so long as the spatial sampling rate is better than Nyquist. The same condition does not usually apply to the temporal part of the source terms, however, which typically must be sampled much faster than Nyquist to obtain acceptable accuracy. In the scheme introduced here, the temporal sampling of the source terms too need only satisfy Nyquist (more than two points-per-period at the highest frequency). This natural extension of the $k$-space PSTD scheme to timevarying sources allows much larger timesteps to be taken without compromising accuracy.

\section{REFERENCES}

[1] T. D. Mast, L.P. Souriau, D.-L.D. Liu, M. Tabei, A.I. Nachman, and R.C. Waag, "A k-space method for large-scale models of wave propagation in tissue," IEEE Trans. Ultrason. Ferroelectr. Freq. Control, vol. 48 , pp.341-354, 2001

[2] M. Tabei, T.D. Mast, and R.C. Waag, "A k-space method for coupled first-order acoustic propagation equations," J. Acoust. Soc. Am., vol. 111, pp. 53-63, 2002.

[3] B.E. Treeby and B.T. Cox, " $k$-Wave: MATLAB toolbox for the simulation and reconstruction of photoacoustic wave fields", J. Biomed. Opt., vol. 15, p. 021314, 2010.

[4] B.E. Treeby, J. Jaros, A.P. Rendell, and B.T. Cox,"Modeling nonlinear ultrasound propagation in heterogeneous media with power law absorption using a k-space pseudospectral method", J. Acoust. Soc. Am., vol. 131, no. 6, pp.4324-4336, 2012.

[5] http://www.k-wave.org

[6] B.E. Treeby, J. Budisky, E.S. Wise, J. Jaros, and B.T. Cox, "Rapid calculation of acoustic fields from arbitrary continuous-wave sources," J. Acoust. Soc. Am., vol. 143, no. 1, pp. 529-537, 2018.

[7] B.E. Treeby, E.S. Wise, and B.T. Cox, "Nonstandard Fourier Pseudospectral Time Domain (PSTD) Schemes for Partial Differential Equations," Commun. Comput. Phys., vol. 24, no. 3, 623-634, 2018.

[8] E.S. Wise, J.L. Robertson, B.T. Cox, and B.E. Treeby, "Staircasefree acoustic sources for grid-based models of wave propagation," Proc. IEEE International Ultrasonics Symposium 2017. doi:10.1109/ULTSYM.2017.8092730.

[9] A. Pierce, Acoustics: An Introduction to its Physical Principles and Applications, Acoust. Soc. Am.,1989. 